\title{
Editorial
}

\section{New Editor-in-Chief's Note: The Past and the Future}

\author{
Rainer H. Straub \\ Laboratory of Experimental Rheumatology and Neuroendocrine Immunology, Department of Internal Medicine, \\ University Hospital, Regensburg, Germany
}

\section{A Look Back}

By studying the history of a scientific journal, we can learn more about the scientific culture of a research field and we can start to understand how it changed and what it is now. Samuel M. McCann ${ }^{\dagger}$ and James M. Lipton ${ }^{\dagger}$ (served: 1994-2001), George P. Chrousos (2002-2006), and Wilson Savino (2007-2021) were the distinguished Editors-in-Chief of Neuroimmunomodulation, giants of the science of neuro-endocrine-immune interrelations. The late Sam McCann wrote in the first Neuroimmunomodulation Editorial in Volume 1, Issue 1, 1994 [1]:

This journal will include reports on all aspects of the rapidly expanding area of research known as neuroimmunomodulation. The field encompasses all of the interactions between the central nervous and the immune systems. In essence, the nervous system alters the functions of the immune system via neural, hormonal, and paracrine actions. Neural and humoral influences from the immune system feedback to the CNS to modulate its influences. This is a subject area, which was in its infancy until the last 5-10 years; there has recently been an explosive increase in our knowledge of these interactions.

This first Editorial explained to readers of Neuroimmunomodulation that there exists a "neuro-endocrine crosstalk with immune cells and vice versa." As such, it looked like an advertisement of a very new field of scientific inquiry. Research in these early years of Neuroimmunomodulation had a strong character of basic science directed toward cell cultures and animal experimentation.
It was physiologic in nature, and the direction was most often from hormone or neuropeptide/neurotransmitter $\rightarrow$ immune cell.

The second Editor-in-Chief, George P. Chrousos starting in 2002, hoped that the journal will be handled fully electronically in the future, while he used fax and emails with little attachments and not much else. He wrote [2]: "I believe that the future of Neuroimmunomodulation is closely linked to the progress of electronic technology and that the journal should brace itself and prepare for a parallel, successful and promising electronic route." Fortunately, all of these things were perfectly established during the last 15 years, thanks to Karger publishers.

In addition, he - as pediatric endocrinologist - wished that the journal gets more open for clinicians inviting "rapid clinical communications," "case reports," "critical round table discussions," "reviews of basic science for the non-specialist," but even "templates for the organization of the delivery of care" for patients [2]. In the 5 years he served for the journal, there was indeed a change of direction toward clinical articles and models of clinical diseases such as multiple sclerosis and experimental encephalomyelitis, autoimmune thyroid disease, systemic lupus erythematosus, inflammatory bowel disease, infectious diseases including parasites, stroke, Parkinson's disease, cancer, psychiatric diseases, and aging research. Stress was another aspect that entered the journal more readily. Neuroimmunomodulation started to demon- karger@karger.com

(c) 2022 S. Karger AG, Basel

www.karger.com/nim
Correspondence to:

Rainer H. Straub, rainer.straub@ukr.de 
strate a basic-to-clinical neuro-endocrine-immune potpourri.

The third Editor-in-Chief, Wilson Savino starting in 2007, very much stimulated special issues with article collections towards a defined clinical research subject. He organized together with guest editors special issues on aging, autoimmune diseases, asthma, infectious diseases, thymus immunology, psychiatric diseases, low dose glucocorticoid therapy, and neurodevelopmental disorders [3-11]. We clearly see how the journal remained open for the clinical aspects of neuro-endocrine-immune interrelations, while also keeping the basic aspects of neuro-immune crosstalk. The direction of research was now going into both directions from brain to periphery and from periphery to brain.

It was also in Wilson Savino's time when new techniques entered the journal like vagus nerve stimulation and its effects on immune function (e.g., $[12,13]$ ), the omics revolution (particularly transcriptomics and genomics, e.g., $[14,15])$, biomarker research (e.g., $[16,17])$, and more recently epigenetic regulation with microRNAs and metanalyses in neuroimmunomodulation (e.g., [1824]). Due to the prevalence of parasitic diseases in Wilson Savino's home continent South America, the role of these diseases for neuro-endocrine-immune alterations became a very special focus (e.g., [25-27]).

After Wilson Savino's term, I feel that the neuro-endocrine-immune research entered every field of biomedical inquiry. This is not a research niche anymore, it is open to every discipline, which reminds me to words of the German Philosopher Arthur Schoppenhauer (17881860), who wrote in 1819 [28]: “...to truth (veritas) ..., only a short celebration of victory is permitted, between the two long periods during which it is attacked as paradoxical or accepted as normal." Today, we recognize that truth goes through three stages: (1) ridicule, (2) opposition, and (3) undisputable acceptance.

After nearly 30 years, we are in stage 3 of this sequence of events because many scientists of all biomedical disciplines are presently including these subjects in their specific work. Now, these formerly Neuroimmunmodulation-specific aspects enter many journals of biomedicine. Since the early times in 1994, the field became a solid discipline of scientific inquiry with many important findings that explained basic physiology, helped to enlighten pathophysiology of many diseases, and aided in establishing new therapies.

\section{A Look at the Future}

Being selected as new Editor-in-Chief is an honor. It is my wish to serve the journal with dedication, responsibility, fairness, excellent stewardship, intuition, and vision. In order to magnetize a broader group of submitting scientists from 2022 onwards, I will institute several changes, which include the following:

1. Aims and scope will be broadened to include the psychoneuroimmunology part of brain-immune research. It is my wish to increase the numbers of papers that focus on behavioral aspects next to neural, endocrine, and immune system interactions in humans and animals. Neuroimmunomodulation already published studies on stress but this needs to be widened to all forms of psycho-neuro-endocrine-immune interrelations. The "P" aspect of psychoneuroimmunology will become more important.

2. The current number of members of the Editorial Board will be increased. Members of the Editorial Board have an active role in ad hoc reviews, proposals of special issues, and selection of pillar articles and breakthrough articles (see also No. 3 \& 5).

3. The journal is open to suggestions of special issues that can be proposed by scientists of the psycho-neuro-endocrine-immune field.

4. In short Editorial manuscripts, I wish to demonstrate so-called pillar articles of psycho-neuro-endocrineimmune interrelations, which really changed - in the past - the understanding of physiology and pathophysiology of diseases. Journal Readers are invited to submit proposals for pillar articles.

5. In addition, short Editorials will present major breakthrough papers in psycho-neuro-endocrine-immune interrelations published in other journals. Journal Readers are invited to submit proposals for breakthrough articles.

6. Sometimes, methods can be highly variable but often people wish a standardized protocol to improve generalizability. Special papers that present standardized protocols or guidelines are welcome. Scientists of the psycho-neuro-endocrine-immune field may send a proposal.

7. Finally, it is our will to streamline the review process. We will have a new team with two managing editors from South America, Daniella A. Mendes Da Cruz (Fundacao Oswaldo Cruz, Rio de Janeiro, Brazil) and from the Asian zone of influence, Raymond ChuenChung Chang (the University of Hong Kong). 
Toward most items in the above list, it is clear that I will not come to a decision on my own. I will rely on Managing Editors, Associate Editors, and the Editorial Board. Behind everything stands the will to find the best papers, to guarantee the quality and novelty of manuscripts, and to foresee trends in this particular research field. Keep in mind: Sometimes an Editor-in-Chief can be wrong in his decisions, nobody is perfect.

In closing, I would like to thank the former Editor-inChief Wilson Savino and the people at Karger Publishers, particularly Marco Casola, for the honor of offering me this important position.

\section{Conflict of Interest Statement}

There is no conflict of interest.

\section{Funding Sources}

Writing of this Editorial has not been supported by specific funding. For longtime funding, the author is grateful to the Deutsche Forschungsgemeinschaft (DFG), the Federal Ministry of Education and Research, the European Union, and the State of Bavaria through local funding by the University Hospital Regensburg.

\section{References}

1 McCann SM. Editorial. Neuroimmunomodulation. 1994;1(1):1.

2 Chrousos GP. New editor-in-chief's note. Neuroimmunomodulation. 2002;10(3):12931.

3 Bauer ME, De la Fuente M. Neuroimmunomodulation and aging. Neuroimmunomodulation. 2008;15:207-9.

4 Perez-Leiros C, Saravia F. Neuroimmune approach to the etiology, pathogenesis and treatment of autoimmune disorders. Neuroimmunomodulation. 2008; 15:1-3.

5 Priftis KN, Chrousos GP. Neuroimmunomodulation in asthma: focus on the hypothalamic-pituitary-adrenal axis. Neuroimmunomodulation. 2009;16:261-2.

6 Montor JM, Bottasso O. Neuroimmunomodulation and infectious diseases. Neuroimmunomodulation. 2009;16:63-4.

7 del Rey A, Kolbe N. Neuroimmunomodulation in health and disease: an integrative biomedical approach. Neuroimmunomodulation. 2011;18:359-60.

8 Savino W, Dardenne M. Neuroendocrine immunology of the thymus. Neuroimmunomodulation. 2011;18:261-2.

9 Teixeira AL, Müller N. Immunology of psychiatric disorders. Neuroimmunomodulation. 2014;21:71.

10 Cutolo M, Chrousos GP, Pincus T. Low dose glucocorticoids in rheumatic diseases. Neuroimmunomodulation. 2015;22:1-2.

11 Gottfried C, Bambini-Junior V. Neuroimmunomodulation in neurodevelopmental disorders. Neuroimmunomodulation. 2018;25: 239-42.

12 Aalbers MW, Klinkenberg S, Rijkers K, Verschuure P, Kessels A, Aldenkamp A, et al. The effects of vagus nerve stimulation on pro- and anti-inflammatory cytokines in children with refractory epilepsy: an exploratory study. Neuroimmunomodulation. 2012;19:352-8.

13 Sánchez-Alemán E, Quintanar-Stephano A, Escobedo G, Campos-Esparza Mdel R, Campos-Rodríguez R, Ventura-Juárez J. Vagoto- my induces deregulation of the inflammatory response during the development of amoebic liver abscess in hamsters. Neuroimmunomodulation. 2015;22:166-80.

14 McGuiness B, Gibney SM, Beumer W, Versnel MA, Sillaber I, Harkin A, et al. Exaggerated increases in microglia proliferation, brain inflammatory response and sickness behaviour upon lipopolysaccharide stimulation in non-obese diabetic mice. Neuroimmunomodulation. 2016;23:137-50.

15 Song H, Zhang X, Chen R, Miao J, Wang L, Cui $\mathrm{L}$, et al. Cortical neuron-derived exosomal microRNA-181c-3p inhibits neuroinflammation by downregulating CXCL1 in astrocytes of a rat model with ischemic brain injury. Neuroimmunomodulation. 2019;26: 217-33.

16 Mastronardi CA, Licinio J, Wong ML. Candidate biomarkers for systemic inflammatory response syndrome and inflammation: a pathway for novel translational therapeutics. Neuroimmunomodulation. 2010;17:359-68.

17 Fábregas BC, Vieira ÉL, Moura AS, Carmo RA, Ávila RE, Abreu MN, et al. A Follow-Up Study of 50 chronic hepatitis $\mathrm{C}$ patients: adiponectin as a resilience biomarker for major depression. Neuroimmunomodulation. 2016; 23:88-97.

18 Cao YL, Dong W, Li YZ, Han W. MicroRNA-653 inhibits thymocyte proliferation and induces thymocyte apoptosis in mice with autoimmune myasthenia gravis by downregulating TRIM9. Neuroimmunomodulation. 2019;26:7-18.

19 Fu H, Cheng Y, Luo H, Rong Z, Li Y, Lu P, et al. Silencing microRNA-155 attenuates kainic acid-induced seizure by inhibiting microglia activation. Neuroimmunomodulation. 2019; 26:67-76.

20 Wu B, Guo Y, Chen Q, Xiong Q, Min S. MicroRNA-193a downregulates HMGB1 to alleviate diabetic neuropathic pain in a mouse model. Neuroimmunomodulation. 2019;26: 250-7.
21 Santucci N, Stampone R, Brandão Ferreira da Silva E, Villar S, Spinelli S, Linhares-Lacerda L, et al. Local regulation of adrenal steroidogenesis: subtle in vitro effects of IL- $1 \beta$ on the human cell line NCI-H295R steroid production along with changes in microRNA profile and orphan nuclear receptors NR4As. Neuroimmunomodulation. 2020; 27:131-41.

22 Huo M, Zheng X, Bai N, Xu R, Yang G, Zhao Z. LncRNA PCAT19 regulates neuropathic pain via regulation of miR-182-5p/JMJD1A in a rat model of chronic constriction injury. Neuroimmunomodulation. 2021;9:1-9.

23 Yuan X, Guo L, Jiang C, Yang X, Huang J. The effect of different administration time and dosage of vitamin $\mathrm{D}$ supplementation in patients with multiple sclerosis: a meta-analysis of randomized controlled trials. Neuroimmunomodulation. 2021;28(3):118-28.

24 Lin J, Zhang W, Wang Z, Zhao F. Interleukin-1 and tnterleukin-6 polymorphisms might influence predisposition to hemorrhagic cerebral vascular diseases: a meta-analysis. Neuroimmunomodulation. 2021;28(4): 222-8.

25 Corrêa-de-Santana E, Paez-Pereda M, Theodoropoulou M, Renner U, Stalla J, Stalla GK, et al. Modulation of growth hormone and prolactin secretion in Trypanosoma cruzi-infected mammosomatotrophic cells. Neuroimmunomodulation. 2009;16:208-12.

26 Pérez AR, Bottasso O, Savino W. The impact of infectious diseases upon neuroendocrine circuits. Neuroimmunomodulation. 2009;16: 96-105.

27 Morales-Montor J, Picazo O, Besedovsky H, Hernández-Bello R, López-Griego L, Becerril-Villanueva E, et al. Helminth infection alters mood and short-term memory as well as levels of neurotransmitters and cytokines in the mouse hippocampus. Neuroimmunomodulation. 2014;21:195-205.

28 Schoppenhauer A. Vorrede zur ersten Auflage (Hrsg.). Leipzig: F. A. Brockhaus; 1819. 